\title{
Biologic mesh for abdominal wall reconstruction
}

This article was published in the following Dove Press journal:

Chronic Wound Care Management and Research

6 November 2014

Number of times this article has been viewed

\section{Kathryn S King' \\ Frank P Albino ${ }^{2}$ \\ Parag Bhanot ${ }^{3}$ \\ 'School of Medicine, Georgetown University Hospital, Washington, DC, USA; ${ }^{2}$ Department of Plastic Surgery, ${ }^{3}$ Department of General Surgery, Georgetown University Hospital, Washington, DC, USA}

Correspondence: Parag Bhanot Department of General Surgery, Georgetown University Hospital, 3800 Reservoir Rd NW, Washington, DC 20007, USA

Tel +I 202444048 I

Email pxb129@gunet.georgetown.edu
Background: Mesh reinforcement significantly decreases rates of recurrence following ventral hernia repair. Historically, biologic mesh was touted as superior in the setting of infection; however, selecting the appropriate mesh for a given clinical scenario is often a matter of debate. The purpose of this review is to highlight a number of the more commonly used biologic mesh products with a review of outcomes from the current literature.

Methods: Outcomes following abdominal wall reconstruction using biologic mesh were reviewed for acellular cadaveric human dermis, cross-linked porcine dermis, non-cross-linked porcine dermis, porcine small intestine submucosa, acellular bovine pericardial, and acellular bovine dermal mesh. Studies with rigorous methods, adequate patient samples, and sufficient follow-up were selected for review.

Results: Hernia recurrence rates following biologic mesh reinforcement vary widely. Porcine small intestine submucosa and bovine pericardium were associated with the lowest hernia recurrence rates. Porcine cross-linked dermal mesh products resulted in higher rates of adhesion formation and lower rates of tissue incorporation compared to non-cross-linked porcine mesh.

Conclusion: Successful ventral hernia repair can be achieved with acceptable complications rates for each of the reviewed mesh products. Biologic meshes have an advantage over synthetic mesh in contaminated wounds but their use may not be cost-effective in all patient populations. Those with and/or at high risk for wound complications may also undergo repair with biologic mesh.

Keywords: biologic mesh, ventral hernia repair, acellular dermal matrix

\section{Introduction}

Ventral hernias may develop following otherwise successful abdominal surgeries, with an estimated incidence of $11 \%$ following laparotomy. ${ }^{1}$ Incisional hernias encompass a breadth of clinical entities ranging from small, clean fascial separations to complete loss of abdominal domain. The surgical approach to abdominal wall reconstruction (AWR) must take into account many variables including defect size, location, etiology, as well as the overall clinical picture in order to develop an appropriate treatment algorithm. These hernias pose difficult reconstructive challenges, often fraught with high recurrence rates and postoperative complications. There is a substantial recurrence rate after ventral herniorrhaphy as well as a high incidence of surgical site occurrences (seroma, hematoma formation) and surgical-site infections. While there is not a successful "one size fits all" approach to AWR, there are treatment modalities, which significantly improve these outcomes.

Reinforcement of a hernia repair with implantable mesh has revolutionized AWR by significantly reducing rates of recurrence compared to suture-only repair. ${ }^{2,3}$ In nearly all 
cases of ventral herniorrhaphy, mesh reinforcement is standard practice to reduce recurrence rates following repair; ${ }^{1,4,5}$ however, there is little consensus on which type of reinforcement is best. ${ }^{6}$ Primary repair without mesh reinforcement is only appropriate in the setting of defects less than $2 \mathrm{~cm}$ while all other defect repairs have been shown to reduce rates of recurrence with mesh reinforcement. ${ }^{6}$ There is growing evidence to support the use of synthetic mesh for the majority of repairs; however, biologic mesh may prove superior in the setting of high-risk or contaminated surgical fields. ${ }^{7}$ Despite this, selecting the appropriate mesh for a given clinical scenario remains a matter of debate. Synthetic or biologic mesh may be chosen based upon a number of overlapping criteria including hernia and patient characteristics. These considerations are beyond the scope of this discussion; however, it is important to consider the specific advantages and limitations of any mesh selected to reinforce the hernia repair.

Biologic mesh, although potentially a viable alternative to synthetic mesh in the properly selected clinical setting, is associated with its own drawbacks. To minimize complications and maximize efficacy, mesh should easily incorporate, resist bowel adhesion formation, demonstrate antimicrobial properties, retain strength to reinforce a fascial repair, and maintain tissue compliance. ${ }^{9-10}$ These characteristics remain the paragon to which all mesh is measured against; however, there is no single product able to provide each of these properties sufficiently. Once the decision to use a biologic mesh is made based upon the clinical milieu of a given ventral hernia, there are a number of reasonable options available for AWR, each with particular strengths and weaknesses. The purpose of this review is not to create an algorithm to determine when a biologic mesh is appropriate but rather to highlight a number of the more commonly used biologic mesh products with a review of outcomes from the current literature.

\section{Biologic mesh}

The most commonly used biologic mesh products are derived from a human, porcine, or bovine source and are processed to remove cellular components while maintaining the collagen extracellular matrix. Removal of cellular components is thought to reduce inflammation and foreign-body responses by the host immune system. The extracellular matrix component is thought not only to provide strength and structure but also to support revascularization and promote integration into the host tissue. The extracellular matrix provides a scaffold for host cells to infiltrate and create a barrier between the mesh and intra-abdominal structures, thereby limiting adhesion formation. Adhesion formation occurs secondary to an inflammatory response that results in capillary permeability and deposition of fibrin. Mesh products that are able to induce mesothelial cell deposition are able to avoid production of multiple adhesions; biologic mesh products have demonstrated better mesothelial cell deposition than synthetic products. ${ }^{8,9}$ While all mesh materials are known to produce adhesions, multiple studies have demonstrated decreased adhesion formation and better tissue integration of biologic mesh products as compared to synthetic mesh products. ${ }^{9-12}$ The revascularization potential of biologic mesh is thought to promote access of host immune cells thereby providing further resistance to persistent infection. This "proinflammatory" property of biologic mesh may prove superior at clearing infections and retarding the progress of biofilm to fulminant infection in the setting of contaminated fields. It is important to note that while biologic mesh materials as a whole have demonstrated better vascularity and less adhesion formation as compared to synthetic mesh products, all biologic mesh products are not the same.

Biologic mesh can be chemically cross-linked, a modification to the structure of the collagen fibrils to resist degradation. Cross-linking of biologic mesh induces strong fibrous tissue formation, which was hypothesized to increase tensile strength and durability, but this is at the expense of revascularization. ${ }^{9,13,14}$ The specific process of chemical crosslinking also plays a role in host response. Lai et al found that chemical cross-linking of bovine pericardium with genipin produced less adhesions with similar cellular regeneration as compared with glutaraldehyde, and genipin is a less cytotoxic agent, suggesting that the chemical used for cross-linking may affect host response to the mesh product. ${ }^{15}$

Biologic mesh can be classified based upon the tissue from which it is derived (dermis, small intestine submucosa, pericardium), the species (human, porcine, bovine), and the presence or absence of cross-linking. Acellular cadaveric human dermis (AlloDerm; LifeCell, Branchburg, NJ, USA), cross-linked porcine dermis (Permacol; Covidien, Mansfield, MA, USA), non-cross-linked porcine dermis (Strattice; LifeCell), porcine small intestine submucosa (Surgisis; Cook Biotech, West Lafayette, IN, USA), acellular bovine pericardial mesh (Tutomesh; Tutogen Medical Gmbh, Neunkirchen am Brand, Germany; Veritas; Synovis Life Technologies, St Paul, MN, USA), and acellular bovine dermal matrix (Surgimend; TEI Bioscience, Inc., Boston, MA, USA) are frequently used mesh materials for reinforcement during AWR. ${ }^{10,17,21}$

\section{Acellular human dermis}

Acellular human dermis (AlloDerm; LifeCell) was initially introduced in the 1990s for use in burn victims and was 
quickly found to be useful for AWR, breast reconstruction, and gynecologic reconstruction. Donated human skin tissue is processed to remove the epidermis, and the resultant acellular tissue matrix is dehydrated for future use as a bioprosthetic mesh. According to the manufacturer, AlloDerm should be properly oriented with the dermal side placed against the tissue that is the most vascularized. The basement membrane side of the matrix is known to inhibit vascular growth and cell penetration and should thus be oriented appropriately to allow for maximal vascularity of the matrix. ${ }^{16}$ Acellular human dermis has proven an excellent bioprosthetic mesh because of its ability to incorporate and revascularize in human tissue. Human acellular dermal matrix has been shown to have better vascular and cellular infiltration in preclinical studies when compared to porcine acellular dermal matrices. ${ }^{17}$ Concerns have been raised, however, as to its ability to maintain tensile strength over time.

One of the primary endpoints for AWR is longevity, and multiple studies have been undertaken to assess the longevity of both synthetic and biologic mesh. Ko et al compared recurrence and complication rates in incisional hernia repair with component separation and intraperitoneal underlay mesh reinforcement using soft mid-weight polypropylene (Prolene Soft Mesh and Proceed Surgical Mesh; Ethicon Inc., Blue Ash, OH, USA) (28 patients) and human acellular dermis (26 patients); they found a much higher recurrence rate among hernias repaired with human acellular dermis as compared to soft polypropylene ( $46 \%$ versus $11 \%$, respectively; mean follow-up 17 months and 16 months, respectively). They also evaluated the recurrence rates among the two groups in patients without contaminated wounds and found that the recurrence rate was still significantly higher in repair with AlloDerm as compared to soft polypropylene (61\% versus $12 \%$, respectively) ${ }^{18}$ suggesting that contamination alone does not account for the higher rate of recurrence. Diaz et al, in a study of 240 patients who underwent repair of a ventral hernia in a compromised field with human acellular dermal matrix, and Lin et al, in a study of 144 patients who underwent AWR with human acellular dermal matrix, reported recurrence rates of $17.1 \%$ and $27.1 \%$, respectively. ${ }^{19,20}$ In a recent study of 251 patients with similar comorbidities who underwent ventral hernia repair, Iacco et al demonstrated a lower recurrence rate (32\% versus $47 \%$ ) and a longer time to recurrence (18 months versus 15 months) in Permacol (124 patients) as compared to AlloDerm (126 patients). ${ }^{21}$ Interestingly, this study also found that obesity and the bridging surgical technique were associated with higher rates of recurrence in both groups while the underlay technique was associated with a lower rate of recurrences. ${ }^{21}$ Similar to other studies, Henry et al also demonstrated a higher recurrence rate when human acellular dermal matrix was used as compared to porcine acellular dermal matrix in a study of 66 patients who underwent AWR with either human or porcine acellular dermal matrix using a variety of surgical techniques. ${ }^{22}$ The etiology of AlloDerm's increased tendency to stretch is related to the higher elastin content compared to nonhuman sources. Acellular human dermis has demonstrated much higher recurrence rates in studies overall as compared to other bioprosthetic mesh products (Table 1).

Although AlloDerm may be associated with higher hernia recurrence rates as compared to other biologic mesh products, it has proven to be a very effective mesh product for use in contaminated environments. In the same 2014 study mentioned previously, Iacco et al reported that AlloDerm was associated with a lower rate of early wound complications (within in the first 30 days of repair) as compared to Permacol (32\% versus $48 \%$ ), although the rate of late wound complications was similar among the two products. ${ }^{21}$ Diaz et al reported surgical-site infections occurred in $40 \%$ of a series of 240 patients who had complex ventral hernias (recurrent ventral hernia in a compromised field) repaired with acellular human dermal matrix. This study, however, failed to find an association between patient comorbidities and surgical-site infection or hernia recurrence. ${ }^{20}$ While acellular human dermis is associated with lower risks of postoperative infections in contaminated fields as compared to synthetic mesh, the overall rates of infection are still high (27\%-40\% in studies examined). ${ }^{19,20}$ Acellular human dermis also appears to have a higher infection rate as compared to other bioprosthetic mesh products (Table 1).

\section{Porcine dermis}

Porcine dermis is chemically cleaned to remove all cellular components while maintaining the extracellular matrix. The acellular dermal matrix can then be cross-linked to form the commercially available Permacol (Covidien), or remain in its non-cross-linked form Strattice (LifeCell). Each of these grafts are used extensively in AWR and demonstrate very different advantages and disadvantages.

\section{Cross-linked}

Permacol is the commercially available cross-linked porcine acellular dermis used in AWR. This product has an advantage over the commercially available human acellular dermal matrix, AlloDerm, in that it does not need to be rehydrated prior to use and can be readily used when removed 
Table I Biologic mesh overview

\begin{tabular}{|c|c|c|c|c|c|c|}
\hline Product & Description & $\begin{array}{l}\text { Overall } \\
\text { complication rates }\end{array}$ & $\begin{array}{l}\text { Recurrence } \\
\text { rates }\end{array}$ & $\begin{array}{l}\text { Rates of } \\
\text { postoperative } \\
\text { infection }\end{array}$ & $\begin{array}{l}\text { Need for } \\
\text { explantation }\end{array}$ & Citation \\
\hline \multirow[t]{5}{*}{ AlloDerm } & Non-cross-linked & $12 \%-15 \%$ & $46 \%$ & - & - & Ko et $\mathrm{al}^{18}$ \\
\hline & human dermis & $30 \%($ early)* & $47 \%$ & - & $9 \%$ & lacco et $\mathrm{al}^{21}$ \\
\hline & & - & $15 \%$ & - & - & Henry et $\mathrm{a}^{22}$ \\
\hline & & - & $17 \%$ & $40 \%$ & - & Diaz et $\mathrm{al}^{20}$ \\
\hline & & - & $27 \%$ & $27 \%$ & - & Lin et $\mathrm{a}^{19}$ \\
\hline \multirow[t]{5}{*}{ Permacol } & Cross-linked porcine & $32 \%$ & $11 \%$ & $7 \%$ & $0 \%$ & Hsu et $\mathrm{al}^{24}$ \\
\hline & dermis & $48 \%$ (early)* & $32 \%$ & - & $11 \%$ & lacco et $\mathrm{al}^{21}$ \\
\hline & & $50 \%$ & $15 \%$ & $10 \%$ & - & Shaikh et $\mathrm{a}^{23}$ \\
\hline & & $53 \%$ & $0 \%$ & $11 \%$ & $0 \%$ & Satterwhite et $\mathrm{a}^{50}$ \\
\hline & & - & $66 \%$ & $37 \%$ & $25 \%$ & Abdelfatah et $\mathrm{a}^{25}$ \\
\hline \multirow[t]{4}{*}{ Strattice } & Non-cross-linked & $24 \%$ & $0 \%$ & $2.4 \%$ & $0 \%$ & Patel et $\mathrm{a}^{27}$ \\
\hline & porcine dermis & $28 \%$ & $0 \%$ & $8 \%$ & - & Cicilioni et $\mathrm{al}^{29}$ \\
\hline & & $31 \%$ & $5 \%$ & $19 \%$ & $0 \%$ & Skipworth et a ${ }^{31}$ \\
\hline & & $57 \% *$ & $43 \%$ & $14 \%$ & $0 \%$ & Zerbib et $\left.a\right|^{30}$ \\
\hline \multirow[t]{4}{*}{ Surgisis } & Non-cross-linked & $25 \%$ & $7 \%$ & $0.7 \%$ & - & Franklin et $\mathrm{al}^{35}$ \\
\hline & porcine small intestine & $25 \%$ & $0 \%$ & $25 \%$ & $25 \%$ & Johnson and \\
\hline & submucosa & $33.3 \%$ & $8 \%$ & $8 \%$ & - & Paquette ${ }^{34}$ \\
\hline & & & & & & Naji et al ${ }^{36}$ \\
\hline Tutomesh & Bovine & $12.5 \%$ & - & $4.2 \%$ & - & van Tuil et $\mathrm{al}^{42}$ \\
\hline \multirow[t]{3}{*}{ and Veritas } & pericardium & $18 \%$ & $0 \%$ & $3 \%$ & - & Gurrado et $\mathrm{al}^{41}$ \\
\hline & & $20.8 \%$ & $8 \%$ & - & - & $D^{\prime} A m b r a$ et $\mathrm{al}^{40}$ \\
\hline & & $23.3 \%$ & $16.7 \%$ & $3 \%$ & $3 \%$ & Limpert et $\mathrm{al}^{39}$ \\
\hline Surgimend & $\begin{array}{l}\text { Bovine acellular } \\
\text { dermal matrix }\end{array}$ & $25.5 \%$ & $3.9 \%$ & $9.8 \%$ & - & Clemens et $\mathrm{al}^{43}$ \\
\hline
\end{tabular}

Notes: Overview of overall complication rate (including recurrence rate when possible), recurrence rate, postoperative-infection rate, and the need for explantation for the five types of bioprosthetic mesh products studied. *Recurrence rate not included in the overall complication rate. (-) No data available.

from the packaging. The cross-linked nature of Permacol is thought to add strength to the mesh product, theoretically resulting in lower rates of hernia recurrence as compared to non-cross-linked biologic mesh products. The cross-linking, however, may lead to delayed revascularization.

Ditzel et al studied the difference in adhesion formation, mesh incorporation, and mesh shrinkage for five different meshes (Prolene, composite mesh, non-cross-linked porcine dermis, cross-linked porcine dermis, and small intestinal mucosa) following intraperitoneal mesh placement in an animal model. Overall, the composite mesh was associated with the least amount of adhesions and good incorporation while the cross-linked porcine dermis was associated with the highest percentage of adhesions within the group of meshes studied. Of the biologic meshes, the non-cross-linked porcine dermis demonstrated the least amount of adhesion formation but was poorly incorporated. ${ }^{9}$ The composite mesh and non-cross-linked porcine dermal mesh products were found to have a visceral layer of mesothelial cells, which likely contributed to decreased adhesion formation. It is hypothesized that cross-linking in general is associated with more adhesion formation than non-cross-linking, indicating that cross-linked mesh products should not be used for intraperitoneal repair.
Postoperative complications and hernia recurrence varies greatly in studies of porcine cross-linked dermal mesh used in AWR. Shaikh et al reported a 35\% complication rate and a $15 \%$ recurrence rate with a median follow-up time of 18 months in a study of 20 patients who underwent AWR with Permacol between 2002 and 2005. ${ }^{23}$ A 2009 study of 28 patients undergoing AWR with Permacol demonstrated a complication rate of $17.8 \%$ and a recurrence rate of $10.7 \%$ with a mean follow-up time of 16 months. ${ }^{24}$ Iacco et al demonstrated a higher complication rate of $48 \%$ and a lower recurrence rate of $32 \%$ in a series of 124 patients who underwent complex hernia repair (recurrent hernia in a compromised field) with Permacol as compared to AlloDerm. ${ }^{21}$ In a study of 56 patients undergoing incisional hernia repair with Permacol, Abdelfatah et al demonstrated an early complication rate of $29 \%$ and a $66 \%$ recurrence rate over 5 years. Forty-nine percent of the cases in this study were classified as clean operations, which could have skewed the complication rate. ${ }^{25}$ In a study of biomechanical properties of different types of porcine mesh (acellular dermal matrix, small intestine submucosa, and pericardium), acellular dermal matrix was found to have the least tensile strength with a $58.3 \%$ postoperative laxity rate and a higher rate of 
adhesions as compared to small intestine submucosa. ${ }^{26}$ While the published recurrence rates associated with cross-linked porcine acellular dermis vary greatly $(0 \%-66 \%$ in studies examined), this graft product seems superior only to acellular human dermis in regards to recurrence (Table 1). The cross-linked nature of this graft does not appear to offer the increased tensile strength intended.

Porcine acellular cross-linked dermal mesh products have been shown to be associated with higher rates of adhesion formation as compared to other mesh products and may not provide the additional tensile strength required to prevent hernia recurrence. Recurrence rates vary widely in the studies presented here, ranging from $0 \%$ to $66 \%$. The lack of longterm follow-up in many of these studies may be skewing the results, and the hernia recurrence rate among repairs with acellular cross-linked dermal matrix may be higher than previously thought. While surgical technique and patient characteristics certainly play a role in recurrence rates and complication rates, data is still lacking to determine the true efficacy of this type of mesh in AWR.

\section{Non-cross-linked}

Porcine dermal matrix is also utilized in the non-cross-linked form (Strattice) for breast reconstruction and AWR. The noncross-linked nature of this mesh is thought to reduce the risk of adhesion development. As demonstrated by Ditzel et al in a 2013 study of both synthetic and biologic mesh, noncross-linked porcine dermis had the least amount of adhesion formation but was poorly incorporated as compared to the other types of mesh. ${ }^{9}$ Another 2013 study compared noncross-linked and cross-linked porcine mesh with attention to the foreign-body response and integration capabilities. Novitsky et al found that while both types of biologic mesh have an inflammatory response, non-cross-linked mesh has the least foreign-body response and the best integration among the meshes studied..$^{14}$ These two studies demonstrate that not cross-linking mesh may lead to less adhesion formation and better vascularization and collagen deposition in human tissue. While more studies are needed to definitively support these findings, in cases where adhesion formation is a concern and biologic mesh is necessary, usage of a noncross-linked material will likely result in fewer adhesive complications then cross-linked mesh.

Multiple studies have been performed to evaluate the complication and recurrence rates associated with non-cross-linked porcine dermal mesh. A 2012 study by Patel et al reported a $24.4 \%$ complication rate in a series of 78 patients who underwent AWR with Strattice. With a mean follow-up time of 474 days, no recurrent hernias were noted. ${ }^{27}$ Parra et al looked at a series of three laparoscopic hernia repairs using Strattice mesh and found no complications or recurrences at 6 months. ${ }^{28}$ Cicilioni et al, however, demonstrated a $28 \%$ complication rate (14-month mean follow-up time) in a series of 25 patients who underwent fascial repair with Strattice mesh following transverse rectus abdominis myocutaneous flap breast reconstruction. ${ }^{29}$ A 2013 study by Zerbib et al demonstrated a $57 \%$ complication rate in a study of 14 patients with an infected abdominal wall defect who underwent repair with Strattice. This study also demonstrated a $43 \%$ recurrence rate with a follow-up time of 13 months. ${ }^{30}$ A 2014 study by Skipworth demonstrated a 5\% recurrence rate (mean follow-up time of 17 months) and a complication rate of $26 \%$ in a study of 58 patients who underwent incisional hernia repair with Strattice mesh. ${ }^{31}$

A wide variety of complication (24\%-57\%) and recurrence rates $(0 \%-43 \%)$ for non-cross-linked porcine acellular dermal mesh exist, with none of the above studies having a follow-up time of 2 years or more; long-term follow-up is key to establishing a true recurrence rate. Non-crosslinked porcine dermal mesh products are clearly efficacious in AWR in limiting postoperative infections - rates of the above-mentioned studies range from $2 \%-19 \%$ - but the true recurrence rate of this mesh product is yet to be established. Few conclusions can be made from the limited data available; however, the studies evaluated do not suggest a benefit of cross-linking in decreasing recurrence rates with porcine acellular dermal grafts. Non-cross-linked acellular porcine dermal matrix has also been demonstrated to be efficacious in the contaminated wounds. A 2012 study by Itani et al was of 80 patients with clean-contaminated, contaminated, or dirty wounds who underwent successful repair with non-cross-linked porcine dermal matrix. At the 24-month follow-up, $28 \%$ of the hernias had recurred..$^{32}$ Non-cross-linked acellular porcine dermal matrix produces less adhesions and superior ultimate tensile strength as compared to cross-linked porcine dermal matrix. ${ }^{33}$ Of the porcine acellular dermal grafts available, the non-cross-linked version appears to be associated with the least complications and lowest recurrence rates, though direct comparisons studies should be undertaken.

\section{Porcine small intestine submucosa}

Acellular porcine small intestine submucosa grafts have been used successfully as arterial/venous grafts. This graft easily promotes revascularization and good incorporation, making it useful in reconstruction. In 1996, Clarke et al performed 
a pilot study to evaluate the use of Surgisis in AWR in an animal model. The study demonstrated complete replacement of the mesh by host tissue at 4 months with significantly fewer adhesions as compared to polypropylene mesh. ${ }^{11}$ Since that time, porcine small intestine submucosa grafts have found a place in AWR. Johnson et al demonstrated the usefulness of Surgisis in contaminated battlefield wounds in a case series of four patients. ${ }^{34}$ Franklin et al reported a $25 \%$ overall complication rate and $7 \%$ recurrence rate in a series of 133 laparoscopic hernia repairs in an infected or potentially contaminated field. ${ }^{35}$ Zhang et al performed a biomechanical analysis of porcine small intestine submucosa in 2011. The report demonstrated better tensile strength when compared to porcine acellular dermal matrix, the highest degree of vascularization when compared to polypropylene and porcine acellular dermal matrix, and the least amount of adhesions. ${ }^{26}$ In the pediatric population, the use of Surgisis (Cook Biotech, West Lafayette, IN, USA) is associated with very few infectious complications and a low recurrence rate. ${ }^{36}$ A recent study by Song et al, demonstrated increased vascularization and mechanical strength with decreased adhesions in porcine small intestine submucosa grafts with a composite scaffold of tenocytes as compared to porcine small intestine submucosa grafts alone. ${ }^{37}$

Overall, porcine small intestine submucosa has proven an effective graft for use in AWR with high tensile strength, good incorporation and vascularization, and a low recurrence rate. The non-cross-linked nature of this mesh product creates an advantage over cross-linked products in regards to complications without affecting the strength of the graft. More large population studies need to be done to fully evaluate this graft; however, the available studies suggest this graft may prove superior to the other porcine grafts and to the human acellular dermal mesh. The studies that have been performed, however, involve few patients and do not necessarily represent the patient population most in need of AWR.

\section{Bovine pericardium and dermal mesh}

Bovine pericardium grafts have been used extensively in the surgical realm including vascular surgery, thoracic surgery, cardiac surgery, and plastic and reconstructive surgery. Tutomesh (Tutogen Medical Gmbh) and Veritas (Synovis Life Technologies) are two types of acellular, cross-linked bovine pericardium that are used in AWR. Bovine pericardium is processed to remove cellular elements while maintaining the cross-linked collagen matrix. A comparison of cross-linked and non-cross-linked bovine pericardial mesh demonstrated better fibrous tissue formation and integration of the crosslinked product as compared to the non-cross-linked product. ${ }^{13}$ Similarly, bovine acellular dermal matrices have become popular in the field of reconstruction, used in AWR, breast reconstruction, and wound care.

Bovine pericardial mesh products have not been extensively studied in AWR and have not been subject to many comparison studies with other biologic mesh products. Animal studies demonstrated less adhesion formation and better tissue integration with acellular bovine pericardium as compared to synthetic mesh or cellular bovine pericardium. ${ }^{15,38}$ Human studies have focused on the use of bovine pericardium in contaminated wounds. A 2009 study of 30 ventral hernia repairs, $61 \%$ of which were complicated by on-going infection or gross contamination, demonstrated a $19 \%$ hernia recurrence rate and a $6.7 \%$ complication rate when repaired with bovine pericardium. ${ }^{39}$ D'Ambra et al studied 48 contaminated abdominal wall repairs with bovine pericardium and found a recurrence rate of $8 \%$ and wound complication rate of $10 \%$ over a mean follow-up of 22 months. ${ }^{40}$ A recent study by Gurrado et al demonstrated a $3 \%$ wound-infection rate and $0 \%$ recurrence rate in a study of contaminated incisional hernia repair with bovine pericardium. Gurrado et al compared the wound-infection and recurrence rates to contaminated incisional hernia repairs with synthetic mesh and suture repair alone and found that bovine pericardium was associated with significantly lower rates of both endpoints. ${ }^{41}$

Based on the limited studies we do have, bovine pericardium has proven beneficial for use in contaminated fields with limited recurrence and wound-complication rates. Bovine pericardium has also proven beneficial in abdominal wall repair in neonates with gastroschisis or omphalocele. A 2006 study of 24 neonates who underwent repair with bovine pericardium demonstrated successful integration of the mesh in all cases but one. ${ }^{42}$ Available studies demonstrate a role for bovine pericardium in multiple types of abdominal wall repair, especially contaminated fields, but more studies need to be done to compare bovine pericardium to other biologic mesh products. Bovine pericardium appears to have a lower complication rate (ranging between $3 \%$ and $10 \%$ in the studies examined $\left.{ }^{39-41}\right)$ and recurrence rate $(0 \%-19 \%)$ than other biologic mesh products (Table 1) but direct comparison studies are not yet available.

\section{Bovine acellular dermal matrix}

Similar to bovine pericardial mesh products, bovine acellular dermal matrix has not yet been aggressively studied 
in the field of AWR. A recent study of 120 patients who underwent a non-bridge inlay AWR with either porcine acellular dermal matrix (69 patients) or bovine acellular dermal matrix (51 patients) demonstrated significantly lower overall complication rates with bovine acellular dermal matrix (44.9\% overall complications in the porcine acellular dermal matrix group versus $25.5 \%$ in the bovine acellular dermal matrix group). This study did not demonstrate a difference in surgical complications $(24.2 \%$ for porcine and $21.6 \%$ for bovine acellular dermal matrix) or rate of hernia recurrence (2.9\% for porcine and $3.9 \%$ for bovine acellular dermal matrix) over a mean follow-up period of 21 months. ${ }^{43}$ This study demonstrates that bovine acellular dermal matrix is comparable to porcine acellular dermal matrices in AWR in regards to hernia recurrence rates and surgical complications but may be superior in regards to overall complications. A 2009 study of five patients who underwent AWR of contaminated wounds demonstrated the potential for the use of bovine acellular dermal matrices in contaminated wounds as only two of five patients demonstrated wound complications. ${ }^{44}$ Butterfield suggest that the cost of bovine acellular dermal matrix makes it a more attractive implant as compared to human acellular dermal matrix in breast reconstruction as both products have similar complication rates while bovine acellular dermal matrix is significantly less expensive. ${ }^{45}$ Overall, bovine acellular dermal matrix is a promising biologic mesh product for AWR and more longterm studies should be done to elucidate its efficacy in limiting surgical complications and hernia recurrence rates.

\section{Discussion}

Mesh reinforcement of ventral hernia repairs not only reduces recurrences but should minimize postoperative complications. The frequently used biologic mesh products discussed above have proven reliable in their durability to reinforce the repair, effective for use even in contaminated fields. ${ }^{46}$ Hernia recurrence rates following biologic mesh reinforcement vary widely with the current literature, supporting rates of $5 \%-66 \%$. This variability may be attributed to surgical technique (bridging technique associated with higher recurrence rates independent of mesh product ${ }^{47}$ ), location of mesh placement, patient characteristics, hernia size, surgical complexity, and length of follow-up with very few studies citing a mean follow-up time greater than 2 years.

Given the high costs associated with biologic mesh, not all reconstructions warrant their use. Furthermore, biologic mesh hernia repairs may still result in wound complication rates of $40 \%$ or higher. ${ }^{20,21,48}$ Specific patient and operative characteristics associated with higher rates of wound complications include diabetes mellitus, smoking, hernia size, and bridging technique. ${ }^{48}$ The question still remains as to which biologic mesh minimizes complications and cost while providing strength and durability to the AWR. Even prosthetic mesh may prove sufficient in the setting of infection if the reconstruction is delayed following successful surgical debridement. ${ }^{22,48,49}$ Use of biologic mesh in AWR should be individualized to each patient, taking into account clinical characteristics, such as wound contamination and risk factors for infection, as well as the patients' desires: infected or potentially contaminated wounds can be repaired with a one-stage hernia repair and reconstruction with potential need to repair in the future, or with multistage debridement and reconstruction. Current indications for utilization of bioprosthetic mesh include complex reconstructions deemed highrisk for wound-healing problems and contaminated wounds (replacement for infected synthetic mesh). Differences between specific biologic mesh products are based on limited findings in the absence of rigorous outcome comparisons between these products. ${ }^{18}$ Overall, the integration of biologic mesh with native tissue confers the advantage of resisting infection and decreasing the need of mesh explantation in a contaminated environment.

\section{Conclusion}

The ideal mesh for use in AWR has yet to be discovered, but vast advances have been made in the art of abdominal wall defect repair. Biologic meshes may have an advantage over synthetic mesh in contaminated wounds, but their use may not be cost-effective in all patient populations. Those with and/or at high risk for wound complications should undergo repair with biologic mesh. Ultimately, more large-scale and long-term studies need to be performed to adequately assess the efficacy of biologic mesh products in AWR.

\section{Disclosure}

No funding was utilized for the preparation of this manuscript. Bhanot is a member of the Speakers Bureau for LifeCell Corporation, Branchburg, NJ, USA. All other authors report no conflicts of interest in this work.

\section{References}

1. Santora TA, Roslyn JJ. Incisional hernia. Surg Clin North Am 1993;73:557-570.

2. Burger JW, Luijendijk RW, Hop WC, Halm JA, Verdaasdonk EG, Jeekel J. Long-term follow-up of a randomized controlled trial of suture versus mesh repair of incisional hernia. Ann Surg. 2004;240:578-583; discussion 583-585. 
3. Luijendijk RW, Hop WC, van den Tol MP, et al. A comparison of suture repair with mesh repair for incisional hernia. $N$ Engl $J$ Med. 2000;343:392-398

4. Lamont PM, Ellis H. Incisional hernia in re-opened abdominal incisions: an overlooked risk factor. Br J Surg. 1988;75:374-376.

5. Luijendijk RW, Lemmen MH, Hop WC, Wereldsma JC. Incisional hernia recurrence following "vest-over-pants" or vertical Mayo repair of primary hernias of the midline. World J Surg. 1997;21:62-65; discussion 66.

6. Ventral Hernia Working Group, Breuing K, Butler CE, et al. Incisional ventral hernias: review of the literature and recommendations regarding the grading and technique of repair. Surgery. 2010;148:544-558.

7. Mariette C, Wind P, Micelli Lupinacci R, et al. Practice patterns in complex ventral hernia repair and place of biological grafts: a national survey among French digestive academic surgeons. J Visc Surg. 2014;151:9-16.

8. Pascual G, Pérez-Köhler B, Rodríguez M, Sotomayor S, Bellón JM. Postimplantation host tissue response and biodegradation of biologic versus polymer meshes implanted in an intraperitoneal position. Surg Endosc. 2014;28:559-569.

9. Ditzel M, Deerenberg EB, Grotenhuis N, et al. Biologic meshes are not superior to synthetic meshes in ventral hernia repair: An experimental study with long-term follow-up evaluation. Surg Endosc. 2013;27:3654-3662.

10. Sikkink CJ, Vries de Reilingh TS, Malyar AW, Jansen JA, Bleichrodt RP, van Goor H. Adhesion formation and reherniation differ between meshes used for abdominal wall reconstruction. Hernia. 2006;10:218-222.

11. Clarke KM, Lantz GC, Salisbury SK, Badylak SF, Hiles MC, Voytik SL. Intestine submucosa and polypropylene mesh for abdominal wall repair in dogs. J Surg Res. 1996;60:107-114.

12. Butler CE, Prieto VG. Reduction of adhesions with composite AlloDerm/ polypropylene mesh implants for abdominal wall reconstruction. Plast Reconstr Surg. 2004;114:464-473.

13. James NL, Poole-Warren LA, Schindhelm K, et al. Comparative evaluation of treated bovine pericardium as a xenograft for hernia repair. Biomaterials. 1991;12:801-809.

14. Novitsky YW, Orenstein SB, Kreutzer DL. Comparative analysis of histopathologic responses to implanted porcine biologic meshes. Hernia. Epub December 27, 2013.

15. Lai PH, Chang Y, Liang HC, Chen SC, Wei HJ, Sung HW. Peritoneal regeneration induced by an acellular bovine pericardial patch in the repair of abdominal wall defects. J Surg Res. 2005;127:85-92.

16. Campbell KT, Burns NK, Ensor J, Butler CE. Metrics of cellular and vascular infiltration of human acellular dermal matrix in ventral hernia repairs. Plast Reconstr Surg. 2012;129:888-896.

17. Campbell KT, Burns NK, Rios CN, Mathur AB, Butler CE. Human versus non-cross-linked porcine acellular dermal matrix used for ventral hernia repair: comparison of in vivo fibrovascular remodeling and mechanical repair strength. Plast Reconstr Surg. 2011;127:2321-2332.

18. Ko JH, Salvay DM, Paul BC, Wang EC, Dumanian GA. Soft polypropylene mesh, but not cadaveric dermis, significantly improves outcomes in midline hernia repairs using the components separation technique. Plast Reconstr Surg. 2009;124:836-847.

19. Lin HJ, Spoerke N, Deveney C, Martindale R. Reconstruction of complex abdominal wall hernias using acellular human dermal matrix: a single institution experience. Am J Surg. 2009;197:599-603; discussion 603.

20. Diaz JJ Jr, Conquest AM, Ferzoco SJ, et al. Multi-institutional experience using human acellular dermal matrix for ventral hernia repair in a compromised surgical field. Arch Surg. 2009;144:209-215.

21. Iacco A, Adeyemo A, Riggs T, Janczyk R. Single institutional experience using biological mesh for abdominal wall reconstruction. Am J Surg. 2014;208(3):480-484.

22. Henry CR, Bradburn E, Moyer KE. Complex abdominal wall reconstruction: an outcomes review. Ann Plast Surg. 2013;71: $266-268$.
23. Shaikh FM, Giri SK, Durrani S, Waldron D, Grace PA. Experience with porcine acellular dermal collagen implant in one-stage tension-free reconstruction of acute and chronic abdominal wall defects. World $J$ Surg. 2007;31:1966-1972; discussion 1973-1974, 1975.

24. Hsu PW, Salgado CJ, Kent K, et al. Evaluation of porcine dermal collagen (Permacol) used in abdominal wall reconstruction. $J$ Plast Reconstr Aesthet Surg. 2009;62:1484-1489.

25. Abdelfatah MM, Rostambeigi N, Podgaetz E, Sarr MG. Long-term outcomes ( $>5$-year follow-up) with porcine acellular dermal matrix $\left(\right.$ Permacol $\left.^{\mathrm{TM}}\right)$ in incisional hernias at risk for infection. Hernia. Epub October 16, 2013.

26. Zhang J, Wang GY, Xiao YP, Fan LY, Wang Q. The biomechanical behavior and host response to porcine-derived small intestine submucosa, pericardium and dermal matrix acellular grafts in a rat abdominal defect model. Biomaterials. 2011;32:7086-7095.

27. Patel KM, Nahabedian MY, Gatti M, Bhanot P. Indications and outcomes following complex abdominal reconstruction with component separation combined with porcine acellular dermal matrix reinforcement. Ann Plast Surg. 2012;69:394-398.

28. Parra MW, Rodas EB, Niravel AA. Laparoscopic repair of potentially contaminated abdominal ventral hernias using a xenograft: a case series. Hernia. 2011;15:575-578.

29. Cicilioni O Jr, Araujo G, Mimbs N, Cox MD. Initial experience with the use of porcine acellular dermal matrix (Strattice) for abdominal wall reinforcement after transverse rectus abdominis myocutaneous flap breast reconstruction. Ann Plast Surg. 2012;68:265-270.

30. Zerbib P, Caiazzo R, Piessen G, et al. Outcome in porcine acellular dermal matrix reinforcement of infected abdominal wall defects: a prospective study. Hernia. Epub September 19, 2013.

31. Skipworth JR, Vyas S, Uppal L, Floyd D, Shankar A. Improved outcomes in the management of high-risk incisional hernias utilizing biological mesh and soft-tissue reconstruction: a single center experience. World J Surg. 2014;38(5):1026-1034.

32. Itani KM, Rosen M, Vargo D, et al. Prospective study of single-stage repair of contaminated hernias using a biologic porcine tissue matrix: the RICH study. Surgery. 2012;152:498-505.

33. Butler CE, Burns NK, Campbell KT, Mathur AB, Jaffari MV, Rios CN. Comparison of cross-linked and non-cross-linked porcine acellular dermal matrices for ventral hernia repair. $J$ Am Coll Surg. 2010;211: 368-376.

34. Johnson EK, Paquette EL. Use of surgisis for abdominal wall reconstruction/closure in battlefield casualties during Operation Iraqi Freedom. Mil Med. 2007;172:1119-1124.

35. Franklin ME Jr, Treviño JM, Portillo G, Vela I, Glass JL, González JJ. The use of porcine small intestinal submucosa as a prosthetic material for laparoscopic hernia repair in infected and potentially contaminated fields: long-term follow-up. Surg Endosc. 2008;22:1941-1946.

36. Naji H, Foley J, Ehren H. Use of surgisis for abdominal wall reconstruction in children with abdominal wall defects. Eur J Pediatr Surg. 2014;24:94-96

37. Song Z, Peng Z, Liu Z, Yang J, Tang R, Gu Y. Reconstruction of abdominal wall musculofascial defects with small intestinal submucosa scaffolds seeded with tenocytes in rats. Tissue Eng Part A. 2013;19:1543-1553.

38. Kapan S, Kapan M, Goksoy E, Karabicak I, Oktar H. Comparison of PTFE, pericardium bovine and fascia lata for repair of incisional hernia in rat model, experimental study. Hernia. 2003;7:39-43.

39. Limpert JN, Desai AR, Kumpf AL, Fallucco MA, Aridge DL. Repair of abdominal wall defects with bovine pericardium. Am J Surg. 2009;198:e60-e65.

40. D’Ambra L, Berti S, Feleppa C, Magistrelli P, Bonfante P, Falco E. Use of bovine pericardium graft for abdominal wall reconstruction in contaminated fields. World J Gastrointest Surg. 2012;4:171-176.

41. Gurrado A, Franco IF, Lissidini G, et al. Impact of pericardium bovine patch (Tutomesh ${ }^{\circledR}$ ) on incisional hernia treatment in contaminated or potentially contaminated fields: retrospective comparative study. Hernia. Epub March 1, 2014. 
42. van Tuil C, Saxena AK, Willital GH. Experience with management of anterior abdominal wall defects using bovine pericard. Hernia. 2006;10:41-47.

43. Clemens MW, Selber JC, Liu J, et al. Bovine versus porcine acellular dermal matrix for complex abdominal wall reconstruction. Plast Reconstr Surg. 2013;131:71-79.

44. Wietfeldt ED, Hassan I, Rakinic J. Utilization of bovine acellular dermal matrix for abdominal wall reconstruction: a retrospective case series. Ostomy Wound Manage. 2009;55:52-56.

45. Butterfield JL. 440 Consecutive immediate, implant-based, singlesurgeon breast reconstruction in 281 patients: a comparison of early outcomes and costs between SurgiMend fetal bovine and AlloDerm human cadaveric acellular dermal matrices. Plast Reconstr Surg. 2013;131:940-951.

46. Eppley BL. Experimental assessment of the revascularization of acellular human dermis for soft-tissue augmentation. Plast Reconstr Surg. 2001;107:757-762.
47. Booth JH, Garvey PB, Baumann DP, et al. Primary fascial closure with mesh reinforcement is superior to bridged mesh repair for abdominal wall reconstruction. J Am Coll Surg. 2013;217:999-1009.

48. Rosen A, Eliassi S, Fors U, et al. A computerised third molar surgery simulator - results of supervision by different professionals. Eur J Dent Educ. 2014;18(2):86-90.

49. Souza JM, Dumanian GA. Routine use of bioprosthetic mesh is not necessary: A retrospective review of 100 consecutive cases of intraabdominal midweight polypropylene mesh for ventral hernia repair. Surgery. 2013;153:393-399.

50. Satterwhite TS, Miri S, Chung C, Spain DA, Lorenz HP, Lee GK. Abdominal wall reconstruction with dual layer cross-linked porcine dermal xenograft: The "Pork Sandwich" herniorraphy. J Plast Reconstr Aesthet Surg. 2012;65:333-341.
Chronic Wound Care Management and Research

\section{Publish your work in this journal}

Chronic Wound Care Management and Research is an international, peer reviewed, open access, online journal publishing original research, reviews, editorials, and commentaries on the causes and management of chronic wounds and the major issues related to chronic wound management. Topics also include chronic wounds as comorbidities to other

\section{Dovepress}

conditions, patient adherence to therapy, and the economic burden of chronic wounds. The manuscript management system is completely online and includes a very quick and fair peer review system, which is all easy to use. Visit http://www.dovepress.com/testimonials.php to read real quotes from published authors.

Submit your manuscript here: http://www.dovepress.com/chronic-wound-care-management-and-research-journal 